\title{
Cognitive Behavioral Therapy For Alleviating The Distress Caused By Tinnitus, Hyperacusis And Misophonia: Current Perspectives
}

This article was published in the following Dove Press journal: Psychology Research and Behavior Management

\author{
Hashir Aazh (D) ${ }^{1-3}$ \\ Michael Landgrebe 4 \\ Ali A Danesh (iD ${ }^{5}$ \\ Brian CJ Moore iD ${ }^{6}$ \\ 'London Tinnitus and Hyperacusis \\ Therapy Specialist Clinic, London, \\ WC2N 5BW, UK; ${ }^{2}$ Guildford Tinnitus \\ and Hyperacusis Therapy Specialist \\ Clinic, Guildford, Surrey, GU2 4RG, UK; \\ ${ }^{3}$ Audiology Department, Royal Surrey \\ County Hospital, Guildford GU2 7XX, \\ UK; ${ }^{4}$ Department of Psychiatry, \\ Psychosomatics and Psychotherapy, Kbo \\ Lech-Mangfall-Hospital Agatharied, \\ Hausham 83734, Germany; ${ }^{5}$ Department \\ of Communication Sciences and \\ Disorders, Florida Atlantic University, \\ Boca Raton, FL, 3343I, USA; \\ ${ }^{6}$ Department of Psychology, University of \\ Cambridge, Cambridge CB2 3EB, UK
}

Correspondence: Hashir Aazh Guildford Tinnitus and Hyperacusis Therapy Specialist Clinic, I Farnham Road, Guildford, Surrey GU2 4RG, UK Email info@hashirtinnitusclinic.com

\begin{abstract}
This article reviews the evidence related to the efficacy of Cognitive Behavioral Therapy (CBT) for alleviating the distress caused by tinnitus, hyperacusis and misophonia. Where available, the review was focused on meta-analyses of randomized controlled trials (RCTs) using either passive control groups (typically waiting list or education only) or active control groups (receiving some other form of treatment). Where data from RCTs were not available, case studies and retrospective studies were reviewed. Analyses were conducted separately for studies of patients with tinnitus, hyperacusis and misophonia. RCTs show that CBT is effective in alleviating the distress caused by tinnitus in comparison to passive control groups and sometimes active control groups. CBT for tinnitus can be effective both in individual and in group settings, whether delivered by psychiatrists, clinical psychologists, or specially trained audiologists. CBT for tinnitus can also be effective when delivered via the internet, when combined with help from audiologists. Usually, CBT does not reduce the loudness of tinnitus but it can improve quality of life. Case studies and some limited RCTs suggest that CBT can also be effective in alleviating the distress caused by hyperacusis and misophonia. However, RCTs with active control groups are currently lacking. There is strong evidence supporting the effectiveness of CBT in alleviating the distress caused by tinnitus. However, it is not yet clear whether CBT is more effective than some other forms of treatment. RCTs with active control groups are needed to establish more clearly the extent to which CBT is effective in alleviating the distress caused by hyperacusis and misophonia.
\end{abstract}

Keywords: tinnitus, hyperacusis, misophonia, cognitive behavioral therapy, tinnitus specialist

\section{Introduction}

\section{Definition And Psychological Consequences Of Tinnitus}

Tinnitus is the perception of sounds in the absence of external acoustic stimulation. Common descriptions of tinnitus are: buzzing noise, high-pitched noise, hissing, whistle, waterfall, grinding wheel, ringing, white noise, wind noise, bubbles, clicks, beeps, static noise and humming. ${ }^{1,2}$ About $20 \%$ of patients are unable to describe what their tinnitus sounds like. ${ }^{1}$ Most people who experience tinnitus also have some degree of hearing loss, but not all people with hearing loss have tinnitus. ${ }^{3-6} \mathrm{~A}$ recent study of patients who attended a Tinnitus and Hyperacusis Therapy Specialist Clinic $(n=445)$ in the UK showed that the perceived loudness of tinnitus was related to the severity of hearing loss, with a regression coefficient, $b=0.022$ 
$(p<0.001){ }^{6}$ The pathophysiology of tinnitus is not fully understood and there is at present no "cure" for tinnitus that results in the tinnitus becoming inaudible.

The emotional and psychological impact of tinnitus has been studied extensively. ${ }^{7-11}$ Aazh and Salvi ${ }^{6}$ showed that tinnitus annoyance was significantly associated with tinnitus loudness (regression coefficient, $b=0.49, p<0.001$ ). Zeman, Koller, et $\mathrm{al}^{12}$ reported that scores on the Tinnitus Handicap Inventory (THI) ${ }^{13}$ were significantly predicted by scores on the Beck Depression Inventory (BDI). ${ }^{14}$ Based on a national survey in Sweden, Hebert, Canlon, et $\mathrm{al}^{15}$ reported that depressive symptoms significantly predicted the severity of the distress caused by tinnitus. Trevis, McLachlan, et $\mathrm{al}^{16}$ reported that depression as measured via the BDI was predicted by tinnitus handicap as measured via the THI (regression coefficient, $b=0.31, p<0.001)$. Aazh and Moore ${ }^{17}$ reported that there was a significant correlation between scores on the THI and suicidal and/or self-harm ideations $(r=0.21, p=0.01)$. Several other studies, reviewed by Durai and Searchfield, ${ }^{18}$ have reported associations between tinnitus and depression. A recent study $(n=621)$ showed that the relationship between tinnitus loudness and depression as measured via the Hospital Anxiety and Depression Scale (depression subscale) (HADS-D) ${ }^{19}$ was fully mediated via tinnitus handicap as measured via the THI, sleep disturbances as measured via the Insomnia Severity Index (ISI), ${ }^{20}$ hyperacusis as measured via the Hyperacusis Questionnaire (HQ), ${ }^{21}$ and anxiety as measured via the Hospital Anxiety and Depression Scale (anxiety subscale) (HADS-A). ${ }^{19}$

These findings are consistent with Cognitive Behavioral Therapy (CBT) conceptual models for tinnitus distress, which are based on the assumption that cognitive processes are involved in creating tinnitus-related distress. ${ }^{22,23}$ CBT models are based on the idea that tinnitus-related distress can be alleviated even when there is no decrease in tinnitus loudness, if the underlying thought processes are successfully modified. ${ }^{24}$

\section{Definition And Psychological Consequences Of Hyperacusis}

Hyperacusis is intolerance of certain everyday sounds that causes significant distress and impairment in social, occupational, recreational, and other day-to-day activities. ${ }^{25}$ The sounds may be perceived as uncomfortably loud, unpleasant, frightening, or painful. ${ }^{26}$ There may be several sub-types of hyperacusis, including pain hyperacusis (the experience of pain in response to certain sounds) and fear hyperacusis (fear evoked by certain sounds), and these may occur singly or in combination. ${ }^{26}$ Tinnitus and hyperacusis often co-occur, as highlighted by Tyler and Conrad Armes. ${ }^{27}$ A study using the Tinnitus Research Initiative (TRI) Database showed that $55 \%$ of patients $(935 / 1713)$ who had tinnitus also had hyperacusis. ${ }^{28}$ The underlying mechanisms of hyperacusis are not understood and currently, like tinnitus, there is no proven "cure" for hyperacusis. ${ }^{29,30}$

Hyperacusis can cause substantial discomfort, inability to access public services, and problems with health and education. ${ }^{29}$ Research studies have shown that over $50 \%$ of patients with hyperacusis also suffer from some form of psychiatric disorders. ${ }^{11,31}$ A recent study in the UK of 139 patients with tinnitus and hyperacusis reported a small but significant correlation between suicidal and self-harm ideations and hyperacusis severity $(r=0.18, p=0.036) .{ }^{17}$ In the absence of a cure, several authors have suggested the use of CBT to alleviate hyperacusis-related distress. ${ }^{32-34}$ Through collaborative work between specialists in audiology and clinical psychology, Aazh and Allott ${ }^{35}$ published a CBT protocol for hyperacusis rehabilitation.

\section{Definition And Psychological Consequences Of Misophonia}

Misophonia is defined as an abnormally strong emotional and behavioral reaction to particular sounds or groups of sounds with a specific pattern and meaning to the patient. ${ }^{36}$ In other words, it is a hatred or dislike of certain sounds, for example, chewing sounds or slurping sounds. Misophonia can have a great impact on the life of the sufferer, leading to disability and social isolation. ${ }^{37} \mathrm{~A}$ consensus paper bringing together experts from a wide range of disciplines suggested using the term "annoyance hyperacusis" for the experience of negative emotional reactions to sound, instead of misophonia. ${ }^{26}$ Most research studies in the field of audiology have not distinguished misophonia from hyperacusis. ${ }^{28,33,38-42}$ Jastreboff and Jastreboff ${ }^{43}$ reported that misophonia is always experienced in cases of severe hyperacusis. Recent studies conducted in the UK suggest that severe hyperacusis is typically characterised by strong across-frequency variations in sensitivity to sound, which is an indication of adverse reactions only to specific sounds, a feature associated with misophonia. ${ }^{44,45}$

In the field of psychiatry, there is a growing debate about whether misophonia is a distinct psychiatric disorder. ${ }^{46,47}$ The most common comorbid psychological disorders for individuals who experience misophonia are 
post-traumatic stress disorder, obsessive-compulsive disorder (OCD) and obsessive-compulsive personality disorder. $^{46,48,49}$ Although misophonia is not listed in any major psychiatric classification system, a few psychiatric clinics have reported treating such patients. ${ }^{46,50-52}$ Systematic studies of misophonia are very limited. Based on the literature it is not clear whether misophonia is a symptom of a distinct psychiatric disorder or a variety of hyperacusis that is often co-morbid with neuropsychiatric disorders. ${ }^{53}$ Several authors have reported promising results using $\mathrm{CBT}$ to help patients with misophonia. ${ }^{50,51,54}$

\section{CBT For Tinnitus, Hyperacusis And Misophonia}

Although it is not clear whether there are causal relationships between anxiety, tinnitus, hyperacusis, and misophonia, there is a growing body of evidence suggesting links between them. ${ }^{11,55}$ In the absence of a cure, treatment of the depressive and anxiety components of the conditions could be beneficial. CBT is a psychological intervention that aims to alleviate anxiety by helping the patient to modify their dysfunctional cognitions, ruminations and safety-seeking behaviors. ${ }^{56,57}$

CBT is based on the use of models to characterize the mechanisms by which tinnitus or certain sounds (in the case of hyperacusis and misophonia) adversely affect the patient's activities or mood. ${ }^{58}$ Examples of CBT models for tinnitus, hyperacusis and misophonia are shown in Figures 1-3, respectively. Note that the three models have some similarities, and as a consequence the general structure of CBT is similar for tinnitus, hyperacusis and misophonia. The CBT models that are proposed in this paper begin with the initial emotional response and physical

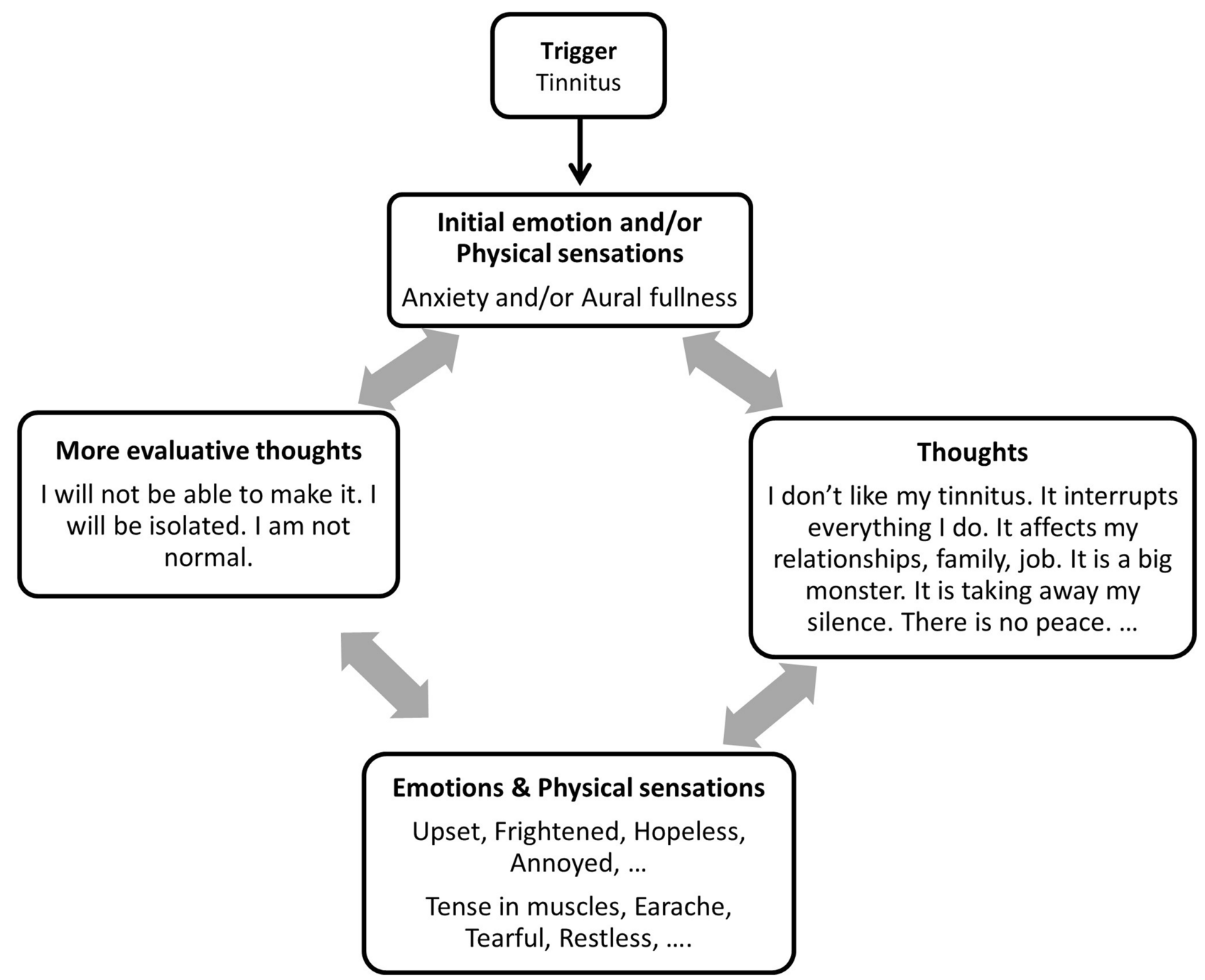

Figure I Case formulation illustrating a model for tinnitus-induced distress. 


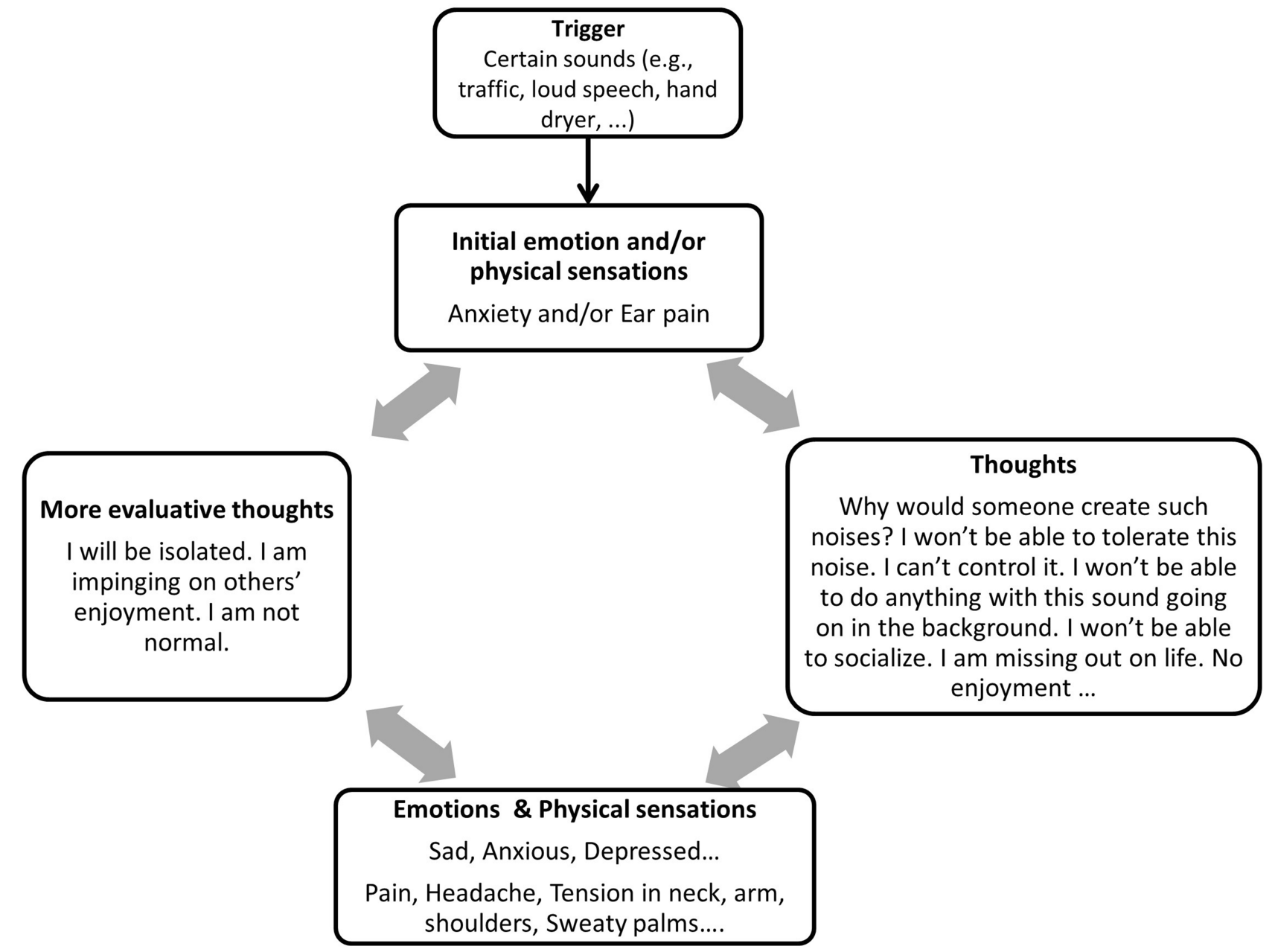

Figure 2 Case formulation illustrating a model for hyperacusis-induced distress.

sensations related to the experience of tinnitus, hyperacusis and misophonia. These initial responses and sensations evoke negative thoughts, leading to further negative emotions and physical sensations, and to more evaluative thoughts and these in turn feed back into the patient's emotional responses and physical sensations. Thus, a vicious cycle is formed, leading to exacerbation of their symptoms. ${ }^{22,23,59}$

The method of delivering CBT for alleviating the distress caused by tinnitus and sound intolerance varies significantly across different settings. Although CBT is typically administered by appropriately trained psychotherapists, psychiatrists and psychologists, the majority of patients with tinnitus and hyperacusis are referred to audiology departments rather than being referred to mental health services, ${ }^{29}$ mainly due the auditory nature of such disorders. In the UK, tinnitus and/or hyperacusis-specific CBT is usually provided by specialized audiologists. In such settings, patients are usually screened for psychological disorders and are referred to mental health services when indicated. ${ }^{11}$ In other countries, a wide range of health and social care professionals may be involved in offering CBT focused on tinnitus and sound intolerance disorders. ${ }^{33,60}$ In some centers, patients with tinnitus and hyperacusis are offered CBT via the internet or self-help materials, with minimal contact with professionals. ${ }^{61,62}$

The next section of this article reviews evidence related to the clinical efficacy of CBT applied in a variety of ways for the alleviation of tinnitus, hyperacusis and misophonia.

\section{The Efficacy Of CBT CBT For Tinnitus}

There are several meta-analyses assessing the overall outcomes of randomized controlled trials (RCTs) of the efficacy of CBT for the alleviation of tinnitus. ${ }^{63-67}$ Most of the RCTS included a "passive" control group, who were 


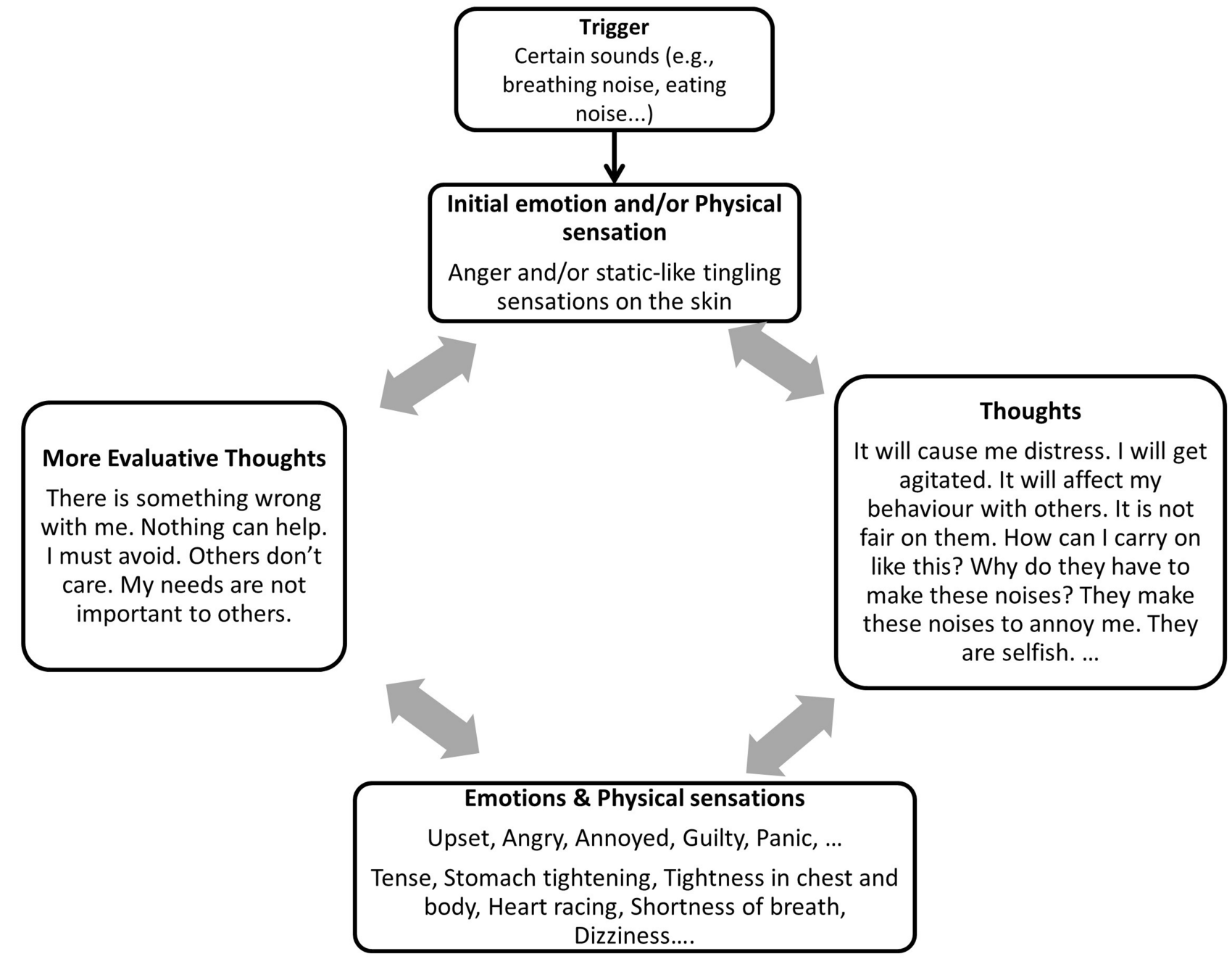

Figure 3 Case formulation illustrating a model for misophonia-induced distress.

on a waiting list, and/or an "active" control group, who received another intervention, such as yoga or education about tinnitus.

Martinez-Devesa, Perera, et $\mathrm{al}^{63}$ included eight RCTs in their analysis. ${ }^{62,68-73}$ There was a tendency for tinnitus loudness to decrease for all groups (treatment, passive control, and active control), but the decrease did not differ significantly across groups. The effect size for CBT versus passive control was only 0.24 ( $95 \%$ confidence interval, CI: -0.02 to 0.51 ) and the effect size for CBT versus active control was only 0.1 ( $95 \%$ confidence interval, CI: -0.22 to 0.42 ). However, tinnitus distress as measured via selfreport questionnaires, the THI and the tinnitus quotient, $\mathrm{TQ},{ }^{74}$ decreased significantly more for the CBT group than for the passive control group (effect size: $0.91,95 \% \mathrm{CI}$ 0.50 to 1.32 ) and than for the active control group (effect size: $0.64,95 \% \mathrm{CI}$ : 0.29 to 1.00 ).
Hesser, Weise, et $\mathrm{al}^{64}$ included 15 RCTs in their analysis. ${ }^{62,68-73,75-81}$ CBT significantly reduced distress and annoyance. The effect sizes for CBT compared with passive control were 0.7 (95\% CI: 0.56 to 0.84 ) for tinnitus distress and 0.35 ( $95 \% \mathrm{CI}: 0.21$ to 0.50$)$ for measures of mood. The effect sizes for CBT compared with active control were $0.44(95 \% \mathrm{CI}: 0.16$ to 0.72$)$ for tinnitus distress and 0.42 (95\% CI: 0.09 to 0.74$)$ for measures of mood. Hesser, Weise, et al ${ }^{64}$ concluded that CBT is effective in reducing the annoyance and distress associated with tinnitus.

Hoare, Kowalkowski, et al ${ }^{66}$ included eight RCTs in their meta-analyses. Studies conducted up to 2010 were included. Two RCTs ${ }^{75,82}$ compared internet-based CBT with control (in one study a waiting list control was used and in the other an internet education with no CBT component was offered to the control group), using the Tinnitus Reaction Questionnaire 
(TRQ). ${ }^{83} \mathrm{CBT}$ had a greater effect than control, with a pooled effect size of 0.38 ( $95 \%$ CI: 0.07 to 0.68 ). Three RCTs compared CBT delivered by mental health professionals with waiting list control using TRQ total scores pre-intervention and post-intervention. ${ }^{68,73,84} \mathrm{CBT}$ had a greater effect than control, with a pooled effect size of 0.54 ( $95 \%$ CI: 0.12 to 0.96$)$. Three RCTs compared CBT delivered by mental health professionals with control using the TQ pre-intervention and post-intervention (the control conditions were waiting list in one study, education and relaxation techniques in one study, and tinnitus retraining therapy in one study). ${ }^{69,71,79}$ CBT had a greater effect than control, with a pooled effect size of 0.64 (95\% CI: 0.31 to 0.98 ). Hoare, Kowalkowski, et $\mathrm{al}^{66}$ concluded that although the overall quality of the RCTs was only moderate, there was sufficient consistency across studies to be confident of the benefit of CBT delivered via mental health professionals for tinnitus intrusiveness.

Nyenhuis, Golm, et al ${ }^{85}$ analyzed 10 RCTs that assessed the efficacy of various forms of self-help CBT comprising internet-delivered CBT, bibliotherapy, self-help book, and self-help manual. ${ }^{61,62,75,80,82,86-89}$ Studies published up to early 2012 were included in the analysis. Most studies included a passive control group that received information only. Nyenhuis, Golm, et $\mathrm{al}^{85}$ reported that self-help CBT significantly reduced tinnitus distress (pooled effect size $=$ 0.54, 95\% CI: 0.30 to 0.77 ) and depression scores (pooled effect size $=0.38,95 \%$ CI: 0.23 to 0.53 ) when compared with passive controls at post-assessment. There was no significant difference in effect sizes between self-help CBT and face-to-face CBT (group treatment and counseling). They concluded that self-help CBT was effective in reducing tinnitus distress. However, they emphasized that although their results suggested comparable effectiveness of self-help and face-to-face CBT, there were only four studies that compared the two forms of treatment and for three of these the CBT was administered in a group rather than individual setting.

Grewal, Spielmann, et al ${ }^{65}$ analyzed eight RCTs that evaluated the effectiveness of CBT and also of Tinnitus Retraining Therapy (TRT) ${ }^{90}$ which has been widely used for the alleviation of tinnitus. Studies published up to 2011 were included in the analysis. ${ }^{62,68-73,79}$ All of the included RCTs were ranked as having a low risk of bias that would significantly alter the results. ${ }^{91}$ Grewal, Spielmann, et al ${ }^{65}$ concluded that both CBT and TRT are effective in reducing tinnitus distress and depressive symptoms as measured via self-report questionnaires. Subjective tinnitus loudness was assessed only for CBT and was not reduced.
The authors concluded that there was insufficient evidence to determine whether CBT or TRT was more effective.

Zenner, Delb, et al ${ }^{67}$ reviewed several RCTs and review studies assessing the effect of CBT on tinnitus distress and quality of life. ${ }^{62,68,69,72,79,92,93}$ They did not report the details of their statistical analysis and their paper was mainly descriptive. Nevertheless, the conclusion was that tinnitus-specific CBT (administered either individually or in groups) is highly effective in alleviating tinnitus distress, depression scores, and improving the quality of life. Two RCTs that were not included in the above-mentioned reviews were conducted by Cima, Maes, et $\mathrm{al}^{94}$ and Maes, Cima, et $\mathrm{al}^{60}$ We briefly discuss their results here. Cima, Maes, et al. ${ }^{94}$ compared two groups. Both received sound therapy and tinnitus education sessions, and one group additionally received 12 weeks of CBT from clinical psychologists. The mean THI score for patients who received CBT improved from 39 (standard deviation, $\mathrm{SD}=23$, $\mathrm{n}=245)$ to $29(\mathrm{SD}=21, \mathrm{n}=175)$ after the full course of treatment, which lasted for 8 months. This change was significantly greater than the change in mean THI score from $39(\mathrm{SD}=23)(\mathrm{n}=247)$ to $34(\mathrm{SD}=25)(\mathrm{n}=161)$ for patients who did not receive CBT. The effect size of the CBT evaluated 12-months post-treatment was 0.24 $(p=0.0009)$ when measured using the Health Utilities Index mark 3 (HUI) ${ }^{95} 0.43(p<0.0001)$ for the TQ, and $0.45(p<0.0001)$ for the THI. Maes, Cima, et $\mathrm{al}^{60}$ assessed the cost-effectiveness of CBT for tinnitus when combined with the usual care (education, sound therapy and counseling, $\mathrm{n}=247)$ compared with usual care alone $(\mathrm{n}=245)$. They calculated the change in quality-adjusted life years (QALY) based on health state utilities measured via the HUI mark 3. Compared with patients receiving the usual care, those who additionally received CBT gained on average 0.015 QALYs (95\% bootstrapped CI: -0.03 to 0.06 ). Maes, Cima, et al ${ }^{60}$ concluded that $\mathrm{CBT}$ combined with usual care is cost-effective compared with usual care only.

There are some recent studies on audiologist-guided internet-based CBT for tinnitus. ${ }^{96,97}$ Beukes, Andersson, et $\mathrm{al}^{96}$ compared eight weeks of audiologist-guided internet-based CBT with two or three face-to-face appointments including education and counseling with no CBT component. The internet-based CBT comprised 16 recommended modules and five optional modules. The content included relaxation, thought analysis, cognitive restructuring, imagery, and exposure techniques. The role of the audiologist was to provide feedback on progress, encouragement, and information about the content of new 
modules. A minimum of 10 mins audiologist time per week was spent on each participant, with more time if required. Seventy-four patients out of 92 who were randomized to the internet-CBT group completed the study. Tinnitus Functional Index (TFI) ${ }^{98}$ scores improved with the treatment for both groups and the difference between the two groups was not statistically significant. In another $\mathrm{RCT}$, Beukes, Baguley, et $\mathrm{al}^{97}$ randomly assigned participants to receive eight weeks of audiologist-guided internet-based CBT (intervention group) or eight weeks of monitoring (control group). Sixty three out of 73 patients who were randomized to the intervention group completed the treatment and all patients randomized to the control group $(n=72)$ completed the control assessments. TFI scores improved significantly more for the intervention group than for the control group. The effect size was 0.7 (95\% CI: 0.4 to 1.0 ).

A recent retrospective study assessed the effectiveness of CBT for tinnitus delivered by audiologists working in the National Health Service in the UK $(n=68){ }^{24}$ The audiologists were specialized in tinnitus and hyperacusis rehabilitation and had attended a Tinnitus and Hyperacusis Therapy Masterclass focusing on 1) psychological and audiological assessment of tinnitus and hyperacusis, ${ }^{11,99}$ 2) client-centered counseling skills, ${ }^{100} 3$ ) special CBT skills required for management of tinnitus and hyperacusis, ${ }^{35,101}$ and 4) motivational interviewing skills. ${ }^{102-104}$ The study was not an RCT as it did not have a control group. THI scores improved significantly post intervention. The effect size for patients who completed the CBT was 1.13. An intention-to-treat analysis, ${ }^{105}$ including those who dropped out in order to avoid any selection bias, also showed significant improvements in THI.

In summary, the meta-analyses and studies reviewed above all indicate that CBT is effective in alleviating the distress caused by tinnitus compared to passive control groups and in some cases compared to active control groups. CBT was usually not found to reduce the loudness of tinnitus. CBT can be effective when delivered in a group setting rather than individually. CBT can also be effective when delivered via the internet, when combined with appropriate help from audiologists. It is unclear whether CBT is more effective than some other active forms of tinnitus treatment, such as TRT.

\section{CBT For Hyperacusis}

The CBT model for hyperacusis (Figure 2) begins with the initial emotions (eg, anxiety) and physical sensations (eg, pain) resulting from the trigger sound. ${ }^{24,59}$ Intolerance to sound as the result of extreme loudness perception or pain are defined as loudness hyperacusis and pain hyperacusis, respectively. ${ }^{26}$ In individuals with hyperacusis-related distress, these initial emotions and sensations are typically followed by a vicious cycle involving negative thoughts leading to further negative emotions and physical sensations, and to more evaluative thoughts, which feed back into the patient's emotional reaction leading to exacerbation of their symptoms. The CBT intervention for hyperacusis distress aims to break the vicious cycle by helping the individual to explore their negative thought processes and to modify them.

To the authors' knowledge, there are only four published studies on the effectiveness of CBT for hyperacusis. Juris, Andersson, et $\mathrm{al}^{33}$ conducted an RCT on the effect of CBT for patients who reported hyperacusis as their main problem. They randomly assigned 30 patients to the CBT group and 32 patients to a passive waiting-list control group. Patients in the CBT group received six sessions of CBT delivered by qualified psychologists, while patients in the waiting-list group received no intervention beyond the assessment of their baseline measures. There were only four drop outs and no intention-to-treat analysis was performed. Scores on the HQ improved significantly for both groups by the end of the study, but the improvement was significantly larger for the CBT group. The difference in pre- and post-treatment scores on the HQ was $8.3(\mathrm{SD}=7)$ for the CBT group and $4.1(\mathrm{SD}=6)$ for the waiting-list group. There was no significant difference between groups in the reduction of the anxiety score as measured via the HADS. A weakness of the study of Juris, Andersson, et $\mathrm{al}^{33}$ was the use of only a passive control group. It would have been better to include an active control group that received some form of treatment involving therapy, but without the specific content of CBT.

In studies of audiologist-guided internet-based $\mathrm{CBT},{ }^{96,97}$ the outcome for hyperacusis was also reported. Beukes, Andersson, et al $^{96}$ compared the effects of eight weeks of audiologist-guided internet-based CBT with two or three face-to-face appointments including education and counseling with no CBT component. The internet-based CBT comprised 16 recommended modules and five optional modules, as described earlier. The improvement in HQ score following treatment did not differ significantly for the two groups. The effect size was $0.16(95 \%$ $\mathrm{CI},-0.26$ to 0.57$)$. In other words, the two interventions were equally effective in reducing hyperacusis handicap. 
In another $\mathrm{RCT}$, Beukes, Baguley, et $\mathrm{al}^{97}$ randomly assigned participants to receive eight weeks of audiologist-guided internet-based CBT (intervention group) or eight weeks of monitoring (control group). Sixty three out of 73 patients who were randomized to the intervention group completed the treatment and all patients randomized to the control group $(n=72)$ completed the control assessments. The audiologist-guided internet-based CBT led to a slightly greater improvement in HQ scores than for the control group, but the effect was only marginally significant. The effect size was $0.3(95 \% \mathrm{CI}=0.0$ to 0.7$)$.

Aazh and Moore $^{24}$ conducted a retrospective study comparing hyperacusis-related distress before and after CBT delivered by audiologists trained in the delivery of CBT $(n=68)$. For the subgroup of patients $(n=14)$ with a diagnosis of hyperacusis based on an HQ score $\geq 22^{44}$ who completed the course of CBT, the mean pre-CBT HQ score was $29.5(\mathrm{SD}=4.4)$ and the mean post-CBT score was $20.8(\mathrm{SD}=6.7)$, an 8.7-point improvement $(p=$ 0.0003 ). Aazh and Moore ${ }^{24}$ also conducted an intentionto-treat analysis, ${ }^{105}$ in order to avoid any selection bias resulting from patients dropping out. The results showed that for the subgroup of patients with hyperacusis based on HQ scores $\geq 22(n=22)$, the mean pre-CBT HQ score was $29.5(\mathrm{SD}=5.1)$ while the mean post-treatment score was $23.2(\mathrm{SD}=8.1)$, a 6.4-point improvement $(p=0.0004)$. Because this study did not have a control group, it is difficult to assess the extent to which the measured improvements were produced specifically by the CBT as opposed to being a more general effect of receiving treatment (placebo effect). ${ }^{106}$ Studies with an RCT design, including long-term follow up, are needed to assess more accurately the efficacy of audiologist-delivered CBT for the management of tinnitus and hyperacusis. However, the results were encouraging, since the effect sizes were medium or large. ${ }^{107}$

In summary, the limited amount of available evidence supports the idea that CBT is effective in reducing hyperacusis handicap as measured via the HQ. However, RCTs with active control groups are needed to establish more conclusively the benefits of CBT in alleviating hyperacusis handicap.

\section{CBT For Misophonia}

The CBT model for misophonia (Figure 3) begins with the initial emotional responses (eg, anger, irritation) and physical sensations (eg, stomach tightening, static-like tingling sensations on the $\operatorname{skin}^{49}$ ) to a specific set of trigger sounds. Typical examples of such sounds are chewing or crunching food, gum popping or chewing, lip smacking, nose sniffing, breathing, clicking sounds, and tapping noises. ${ }^{26,108}$ In individuals with misophonia-related distress, these initial emotional reactions and physical sensations are typically followed by a vicious cycle involving negative thoughts leading to further negative emotions and physical sensations, and to more evaluative thoughts that feed back into the patient's emotional reaction, leading to exacerbation of their negative feelings about the trigger sounds. In addition, the observation of specific movements or anticipation of the trigger sounds can trigger intense aversive responses associated with feelings of distress, disgust, irritability, and anger, which lead to increased focus on the sound source. ${ }^{46,108}$ The CBT intervention for misophonia-related distress aims to break the vicious cycle by helping the individual to explore their negative thought processes and to modify them. Unfortunately, there appear to be no published RCTs evaluating the effectiveness of CBT in alleviating the distress caused by misophonia. Therefore, the review below is based on case studies.

Reid, Guzick, et al ${ }^{109}$ reported a case of an adolescent with OCD and misophonia. The treatment involved CBT combined with exposure to and response prevention for trigger sounds. Misophonia symptoms were significantly reduced during treatment, but the contribution of the CBT to this improvement is not clear. There are two case reports of a counter-conditioning method called "Neural Repatterning" combined with relaxation therapy. ${ }^{110,111}$ Misophonia symptoms were reduced following treatment. It should be noted that the treatment here involved behavioral therapy, and did not include the specific content of CBT.

There are two case reports on the effect of CBT on misophonia. ${ }^{50,51}$ Bernstein, Angell, et al. ${ }^{50}$ reported a case of a 19-year old female who was intensely irritated by other people slurping, swallowing, and chewing. Her self-report scores on the Beck depression and anxiety questionnaires $^{14}$ were within the normal range and no Axis I disorders (all psychological diagnostic categories except mental retardation and personality disorder) were identified using the structured clinical interview of DSM-IV. ${ }^{112}$ The patient received six sessions of CBT and showed improvement in her misophonia symptoms. The patient still found trigger noises to be unpleasant, but these no longer interrupted her day-to-day activities and did not affect her mood. 
McGuire, $\mathrm{Wu}$, et $\mathrm{al}^{51}$ reported two cases of teenagers with irritability and anxiety produced by other people slurping, swallowing, chewing, sniffing, tapping, and breathing. The teenagers did not seem to have any psychiatric co-morbidity. They received between 10 and 18 CBT sessions focused on their sound-annoyance symptoms. The symptoms following the treatment were improved, as shown by reductions in scores for self-report questionnaires. The parents of the teenagers reported that the trigger sounds no longer disrupted family functioning.

Schroder, Vulink, et $\mathrm{al}^{54}$ reported the results of CBT for 90 patients with misophonia (mean age $=36$ years, SD $=12$ years). Over $70 \%$ of the patients were female. The patients were assessed and diagnosed by psychiatrists who had experience with OCD and related disorders. The CBT sessions were given weekly for seven to eight weeks in a group setting. Each session lasted for four hours and comprised: psychological education, psychomotor therapy, counter-conditioning, and counseling. There were four to nine patients per group and the CBT was delivered by clinical psychologists with extensive experience in CBT for OCD. The Amsterdam Misophonia Scale (A-MISO$S)^{46}$ was administered by a trained psychiatry resident at three time points, comprising: T0 (initial assessment), T1 (prior to the start of the CBT), which was on average 29 weeks ( $\mathrm{SD}=16$ weeks) after the initial assessment, and T2 (end of the treatment). All patients were added to a waiting list after the initial assessment, which led to the time lapse between T0 and T1. The mean A-MISO-S score was 13.6 $(\mathrm{SD}=2.9)$ at $\mathrm{T} 0$ and remained unchanged at $\mathrm{T} 1$. The mean A-MISO-S score was reduced (improved) by 4.5 points $(\mathrm{SD}=3.5)$ at $\mathrm{T} 2 \quad(p<0.001)$. This suggests that $\mathrm{CBT}$ delivered in group setting is helpful for patients with misophonia.

In summary, the limited available evidence suggests that CBT is effective in treating misophonia. However, there is a lack of RCTs. Future studies should include one or more control groups, preferably including an active control group, randomization of patients to groups, and the use of validated misophonia outcome measures.

\section{Patients' Views Of The Effectiveness Of CBT}

For CBT to be effective, and for the course of treatment to be completed, the patient must be motivated appropriately. Patients with tinnitus and hyperacusis often use internetbased sites to find out more about their condition and to explore the treatment options available to them. They also may meet other sufferers via self-help groups. The opinion of others who have received various forms of treatment can be important in the decision of a patient to seek help and in the decision about whether to undergo CBT, if offered. Therefore, it is important to ascertain whether or not CBT is viewed as acceptable and effective by patients who have received CBT. Aazh, Moore, et $\mathrm{al}^{25}$ assessed patients' views about the effectiveness of audiologistdelivered CBT for the management of tinnitus and hyperacusis. Of 77 patients who received audiologist-delivered $\mathrm{CBT}$ as a part of their tinnitus and/or hyperacusis rehabilitation, $65 \%$ ranked its effectiveness as $5 / 5$ (where 1 is no effect and 5 is very effective) and $29 \%$ ranked it as $4 / 5$. Patients' feedback about audiologist-delivered CBT did not differ significantly between patients with tinnitus only and those with hyperacusis (with or without tinnitus). Thus, audiologist-delivered CBT was viewed very positively by the great majority of patients who received it for the treatment of tinnitus and hyperacusis.

\section{Conclusion}

There is strong evidence from RCTs supporting the effectiveness of CBT in alleviating the distress caused by tinnitus in comparison to passive control groups and sometimes active control groups. CBT for tinnitus can be effective both in individual and in group settings, whether delivered by psychiatrists, clinical psychologists, or specially trained audiologists. CBT for tinnitus can also be effective when delivered via the internet, when combined with help from audiologists. CBT does not usually reduce the loudness of tinnitus. Further RCTs are needed to establish whether CBT is more effective than some other forms of psychological interventions.

Case studies and some limited RCTs suggest that CBT can also be effective in alleviating the distress caused by hyperacusis and misophonia. However, RCTs with active control groups are needed to establish the effectiveness of CBT for hyperacusis and misophonia.

\section{Disclosure}

The authors report no conflicts of interest in this study.

\section{References}

1. Aazh H, Moore BCJ, Glasberg BR. Simplified form of tinnitus retraining therapy in adults: a retrospective study. BMC Ear Nose Throat Disord. 2008;8:1-7. doi:10.1186/1472-6815-8-7 
2. Tyler RS, Conrad-Armes D. Tinnitus pitch: a comparison of three measurement methods. Br J Audiol. 1983;17:101-107.

3. Nicolas-Puel C, Faulconbridge RL, Guitton $M$, et al. Characteristics of tinnitus and etiology of associated hearing loss: a study of 123 patients. Int Tinnitus J. 2002;8:37-44.

4. Tyler RS, Baker LJ. Difficulties experienced by tinnitus sufferers. $J$ Speech Hear Disord. 1983;48:150-154. doi:10.1044/jshd.4802.150

5. Mazurek B, Olze H, Haupt H, Szczepek AJ. The more the worse: the grade of noise-induced hearing loss associates with the severity of tinnitus. Int J Environ Res Public Health. 2010;7:3071-3079. doi:10.3390/ijerph7083071

6. Aazh H, Salvi R. The relationship between severity of hearing loss and subjective tinnitus loudness among patients seen in a specialist tinnitus and hyperacusis therapy clinic in UK. J Am Acad Audiol. 2018.

7. Bartels H, Middel BL, van der Laan BF, Staal MJ, Albers FW. The additive effect of co-occurring anxiety and depression on health status, quality of life and coping strategies in help-seeking tinnitus sufferers. Ear Hear. 2008;29:947-956. doi:10.1097/AUD.0b013e $3181888 \mathrm{f} 83$

8. Andersson G, Carlbring P, Kaldo V, Strom L. Screening of psychiatric disorders via the Internet. A pilot study with tinnitus patients. Nord J Psychiatry. 2004;58:287-291. doi:10.1080/ 08039480410005792

9. Mathias K, Mezzasalma M, Nardi A. Prevalence of panic disorder in patients with tinnitus [in Portuguese]. Rev Psiquiatr Clín. 2011;38:139-142. doi:10.1590/S0101-60832011000400005

10. Adoga AA, Adoga AS, Obindo JT. Tinnitus and the prevalence of co-morbid psychological stress. Niger J Med. 2008;17:95-97.

11. Aazh H, Moore BCJ. Usefulness of self-report questionnaires for psychological assessment of patients with tinnitus and hyperacusis and patients' views of the questionnaires. Int $J$ Audiol. 2017;56:489-498. doi:10.1080/14992027.2017.1298850

12. Zeman F, Koller M, Figueiredo R, et al. Tinnitus handicap inventory for evaluating treatment effects: which changes are clinically relevant? Otolaryngol Head Neck Surg. 2011;145:282-287. doi:10.1177/0194599811403882

13. Newman CW, Jacobson GP, Spitzer JB. Development of the tinnitus handicap inventory. Arch Otolaryngol Head Neck Surg. 1996;122:143-148. doi:10.1001/archotol.1996.01890140029007

14. Beck AT, Steer RA. Internal consistencies of the original and revised beck depression inventory. J Clin Psychol. 1984;40:13651367. doi:10.1002/1097-4679(198411)40:6<1365::aid-jclp2270400 615>3.0.co;2-d

15. Hebert S, Canlon B, Hasson D, et al. Tinnitus severity is reduced with reduction of depressive mood-a prospective population study in Sweden. PLoS One. 2012;7:e37733. doi:10.1371/journal.pone. 0037733

16. Trevis KJ, McLachlan NM, Wilson SJ. Psychological mediators of chronic tinnitus: the critical role of depression. J Affect Disord. 2016;204:234-240. doi:10.1016/j.jad.2016.06.055

17. Aazh H, Moore BCJ. Thoughts about suicide and self-harm in patients with tinnitus and hyperacusis. $J$ Am Acad Audiol. 2018;29:255-261. doi:10.3766/jaaa.16181

18. Durai M, Searchfield G. Anxiety and depression, personality traits relevant to tinnitus: a scoping review. Int J Audiol. 2016;55:605615. doi:10.1080/14992027.2016.1198966

19. Zigmond AS, Snaith RP. The hospital anxiety and depression scale. Acta Psychiatr Scand. 1983;67:361-370. doi:10.1111/j.1600-0447. 1983.tb09716.x

20. Bastien $\mathrm{CH}$, Vallieres A, Morin CM. Validation of the insomnia severity index as an outcome measure for insomnia research. Sleep Med. 2001;2:297-307.

21. Khalfa S, Dubal S, Veuillet E, et al. Psychometric normalization of a hyperacusis questionnaire. ORL J Otorhinolaryngol Relat Spec. 2002;64:436-442. doi:10.1159/000067570
22. McKenna L, Handscomb L, Hoare DJ, Hall DA. A scientific cognitive-behavioral model of tinnitus: novel conceptualizations of tinnitus distress. Front Neurol. 2014;5:196. doi:10.3389/fneur. 2014.00196

23. Hallam RS, Jakes SC, Hinchcliffe R. Cognitive variables in tinnitus annoyance. Br J Clin Psychol. 1988;27(Pt 3):213-222.

24. Aazh H, Moore BCJ. Effectiveness of audiologist-delivered cognitive behavioral therapy for tinnitus and hyperacusis rehabilitation: outcomes for patients treated in routine practice. Am J Audiol. 2018;27:547-558. doi:10.1044/2018 AJA-17-0096

25. Aazh H, Moore BCJ, Lammaing $\bar{K}$, Cropley M. Tinnitus and hyperacusis therapy in a UK National Health Service audiology department: patients' evaluations of the effectiveness of treatments. Int J Audiol. 2016;55:514-522. doi:10.1080/14992027.2016.1178 400

26. Tyler RS, Pienkowski M, Rojas Roncancio E, et al. A review of hyperacusis and future directions: part I. definitions and manifestations. Am J Audiol. 2014;23:402-419. doi:10.1044/2014_AJA-140010

27. Tyler RS, Conrad Armes D. The determination of tinnitus loudness considering the effects of recruitment. $J$ Speech Hear Res. 1983;26:59-72. doi:10.1044/jshr.2601.59

28. Schecklmann M, Landgrebe M, Langguth B. TRI database study group. Phenotypic characteristics of hyperacusis in tinnitus. PLoS One. 2014;9:e86944. doi:10.1371/journal.pone.0086944

29. Aazh H, McFerran D, Salvi R, et al. Insights from the First International Conference on Hyperacusis: causes, evaluation, diagnosis and treatment. Noise Health. 2014;16:123-126. doi:10.4103/ 1463-1741.132100

30. Baguley DM. Hyperacusis. J R Soc Med. 2003;96:582-585. doi: $10.1258 /$ jrsm.96.12.582

31. Juris L, Andersson G, Larsen HC, Ekselius L. Psychiatric comorbidity and personality traits in patients with hyperacusis. Int J Audiol. 2013;52:230-235. doi:10.3109/14992027.2012.743 043

32. Baguley D, Andersson G. Hyperacusis: Mechanisms, Diagnosis, and Therapies. San Diego: Plural Publishing Inc; 2007.

33. Juris L, Andersson G, Larsen HC, Ekselius L. Cognitive behaviour therapy for hyperacusis: a randomized controlled trial. Behav Res Ther. 2014;54c:30-37. doi:10.1016/j.brat.2014.01.001

34. Pienkowski M, Tyler RS, Roncancio ER, et al. A review of hyperacusis and future directions: part II. measurement, mechanisms, and treatment. Am J Audiol. 2014;23:420-436. doi:10.1044/2014 AJA-13-0037

35. Aazh H, Allott R. Cognitive behavioural therapy in management of hyperacusis: a narrative review and clinical implementation. Auditory Vestibular Res. 2016;25:63-74.

36. Jastreboff PJ, Jastreboff MM. Tinnitus retraining therapy for patients with tinnitus and decreased sound tolerance. Otolaryngol Clin North Am. 2003;36:321-336.

37. Porcaro CK, Alavi E, Gollery T, Danesh AA. Misophonia: awareness and responsiveness in academia. J Postsecond Educ Disabil. 2019;32:108-118.

38. Sheldrake J, Diehl PU, Schaette R. Audiometric characteristics of hyperacusis patients. Front Neurol. 2015;6:105. doi:10.3389/ fneur.2015.00105

39. Fackrell K, Fearnley C, Hoare DJ, Sereda M. Hyperacusis questionnaire as a tool for measuring hypersensitivity to sound in a tinnitus research population. Biomed Res Int. 2015;2015:290425. doi:10.1155/2015/290425

40. Schecklmann M, Lehner A, Schlee W, et al. Validation of screening questions for hyperacusis in chronic tinnitus. Biomed Res Int. 2015;2015:191479. doi:10.1155/2015/191479

41. Andersson G, Lindvall N, Hursti T, Carlbring P. Hypersensitivity to sound (hyperacusis): a prevalence study conducted via the Internet and post. Int J Audiol. 2002;41:545-554. 
42. Zaugg TL, Thielman EJ, Griest S, Henry JA. Subjective reports of trouble tolerating sound in daily life versus loudness discomfort levels. Am J Audiol. 2016;25:359-363. doi:10.1044/2016_AJA-150034

43. Jastreboff PJ, Jastreboff MM. Decreased sound tolerance: hyperacusis, misophonia, diplacousis, and polyacousis. Handb Clin Neurol. 2015;129:375-387. doi:10.1016/B978-0-444-62630-1.00021-4

44. Aazh H, Moore BCJ. Factors related to uncomfortable loudness levels for patients seen in a tinnitus and hyperacusis clinic. Int $J$ Audiol. 2017;56:793-800. doi:10.1080/14992027.2017.1335888

45. Aazh H, Moore BCJ. Prevalence and characteristics of patients with severe hyperacusis among patients seen in a tinnitus and hyperacusis clinic. J Am Acad Audiol. 2018;29:626-633. doi:10. 3766/jaaa.17015

46. Schroder A, Vulink N, Denys D. Misophonia: diagnostic criteria for a new psychiatric disorder. PLoS One. 2013;8:e54706. doi:10. 1371/journal.pone.0054706

47. Taylor S. Misophonia: a new mental disorder? Med Hypotheses. 2017;103:109-117. doi:10.1016/j.mehy.2017.05.003

48. Erfanian M, Kartsonaki C, Keshavarz A. Misophonia and comorbid psychiatric symptoms: a preliminary study of clinical findings. Nord J Psychiatry. 2019;73:219-228. doi:10.1080/08039488.2019.1609086

49. Rouw R, Erfanian M. A large-scale study of misophonia. J Clin Psychol. 2017.

50. Bernstein RE, Angell KL, Dehle CM. A brief course of cognitive behavioural therapy for the treatment of misophonia: a case example. Cognit Behav Therapist. 2013;6:e10. doi:10.1017/S1754470X1300 0172

51. McGuire JF, Wu MS, Storch EA. Cognitive-behavioral therapy for 2 youths with misophonia. J Clin Psychiatry. 2015;76:573-574. doi:10.4088/JCP.14cr09343

52. Webber TA, Johnson PL, Storch EA. Pediatric misophonia with comorbid obsessive-compulsive spectrum disorders. Gen Hosp Psychiatry. 2014;36:231.e1-2. doi:10.1016/j.genhosppsych.2013.10.018

53. Cavanna AE. What is misophonia and how can we treat it? Expert Rev Neurother. 2014;14:357-359. doi:10.1586/14737175.2014.892418

54. Schroder AE, Vulink NC, van Loon AJ, Denys DA. Cognitive behavioral therapy is effective in misophonia: an open trial. $J$ Affect Disord. 2017;217:289-294. doi:10.1016/j.jad.2017.04.017

55. Edelstein M, Brang D, Rouw R, Ramachandran VS. Misophonia: physiological investigations and case descriptions. Front Hum Neurosci. 2013;7:296. doi:10.3389/fnhum.2013.00296

56. Beck AT. Cognitive Therapy and the Emotional Disorders. New York: International Universities Press; 1976.

57. Clark DA, Beck AT, Alford BA. Scientific Foundations of Cognitive Theory and Therapy of Depression. New York: Wiley; 1999.

58. Muran JC. A reformulation of the ABC model in cognitive psychotherapies: implications for assessment and treatment. Clin Psychol Rev. 1991;11:399-418. doi:10.1016/0272-7358(91)90115-B

59. Aazh H, Moore BCJ. Proportion and characteristics of patients who were offered, enrolled in and completed audiologist-delivered cognitive behavioural therapy for tinnitus and hyperacusis rehabilitation in a specialist UK clinic. Int $J$ Audiol. 2018;57:415-425. doi:10.1080/14992027.2018.1431405

60. Maes IH, Cima RF, Anteunis LJ, et al. Cost-effectiveness of specialized treatment based on cognitive behavioral therapy versus usual care for tinnitus. Otol Neurotol. 2014;35:787-795. doi:10. 1097/MAO.0000000000000331

61. Jasper K, Weise C, Conrad I, et al. Internet-based guided self-help versus group cognitive behavioral therapy for chronic tinnitus: a randomized controlled trial. Psychother Psychosom. 2014;83:234 246. doi:10.1159/000360705

62. Kaldo V, Cars S, Rahnert M, Larsen HC, Andersson G. Use of a self-help book with weekly therapist contact to reduce tinnitus distress: a randomized controlled trial. J Psychosom Res. 2007; 63:195-202. doi:10.1016/j.jpsychores.2007.04.007
63. Martinez-Devesa P, Perera R, Theodoulou M, Waddell A. Cognitive behavioural therapy for tinnitus. Cochrane Database Syst Rev. 2010;8:Cd005233.

64. Hesser H, Weise C, Westin VZ, Andersson G. A systematic review and meta-analysis of randomized controlled trials of cognitivebehavioral therapy for tinnitus distress. Clin Psychol Rev. 2011;31:545-553. doi:10.1016/j.cpr.2010.12.006

65. Grewal R, Spielmann PM, Jones SE, Hussain SS. Clinical efficacy of tinnitus retraining therapy and cognitive behavioural therapy in the treatment of subjective tinnitus: a systematic review. J Laryngol Otol. 2014;128:1028-1033. doi:10.1017/S0022215114002849

66. Hoare DJ, Kowalkowski VL, Kang S, Hall DA. Systematic review and meta-analyses of randomized controlled trials examining tinnitus management. Laryngoscope. 2011;121:1555-1564. doi:10.1002/lary.21825

67. Zenner HP, Delb W, Kroner-Herwig B, et al. A multidisciplinary systematic review of the treatment for chronic idiopathic tinnitus. Eur Arch Otorhinolaryngol. 2017;274:2079-2091. doi:10.1007/ s00405-016-4401-y

68. Andersson G, Porsaeus D, Wiklund M, Kaldo V, Larsen HC. Treatment of tinnitus in the elderly: a controlled trial of cognitive behavior therapy. Int J Audiol. 2005;44:671-675.

69. Kröner-Herwig B, Frenzel A, Fritsche G, Schilkowsky G, Esser G The management of chronic tinnitus: comparison of an outpatient cognitive-behavioral group training to minimal-contact interventions. J Psychosom Res. 2003;54:381-389. doi:10.1016/s0022-39 99(02)00400-2

70. Kröner-Herwig B, Hebing G, van Rijn-Kalkmann U, et al. The management of chronic tinnitus - comparison of a cognitive-behavioural group training with yoga. J Psychosom Res. 1995;39:153165. doi:10.1016/0022-3999(94)00098-p

71. Rief W, Weise C, Kley N, Martin A. Psychophysiologic treatment of chronic tinnitus: a randomized clinical trial. Psychosom Med. 2005;67:833-838. doi:10.1097/01.psy.0000174174.38908.c6

72. Weise C, Heinecke K, Rief W. Biofeedback-based behavioral treatment for chronic tinnitus: results of a randomized controlled trial. $J$ Consult Clin Psychol. 2008;76:1046-1057. doi:10.1037/a0013811

73. Henry J, Wilson P. The psychological management of tinnitus: comparison of a combined cognitive educational program, education alone and a waiting-list control. Int Tinnitus J. 1996;2:9-20.

74. Goebel G, Hiller W. [The tinnitus questionnaire. A standard instrument for grading the degree of tinnitus. Results of a multicenter study with the tinnitus questionnaire]. Hno. 1994;42:166-172.

75. Andersson G, Strömgren T, Ström L. Randomised controlled trial of Internet based cognitive behavior therapy for distress associated with tinnitus. Psychosom Med. 2002;64:810-816. doi:10.1097/01. psy.0000031577.42041.f8

76. Lindberg P, Scott B, Melin L, Lyttkens L. The psychological treatment of tinnitus: an experimental evaluation. Behav Res Ther. 1989;27:593-603. doi:10.1016/0005-7967(89)90143-5

77. Scott B, Lindberg P, Lyttkens L, Melin L. Psychological treatment of tinnitus. An experimental group study. Scand Audiol. 1985;14:223-230.

78. Seydel C, Haupt H, Szczepek AJ, Klapp BF, Mazurek B. Longterm improvement in tinnitus after modified tinnitus retraining therapy enhanced by a variety of psychological approaches. Audiol Neurotol. 2010;15:69-80. doi:10.1159/000231632

79. Zachriat C, Kroner-Herwig B. Treating chronic tinnitus: comparison of cognitive-behavioural and habituation-based treatments. Cogn Behav Ther. 2004;33:187-198. doi:10.1080/16506070410029568

80. Hesser H, Gustafsson T, Lunden C, et al. A randomized controlled trial of Internet-delivered cognitive behavior therapy and acceptance and commitment therapy in the treatment of tinnitus. $J$ Consult Clin Psychol. 2012;80:649-661. doi:10.1037/a0027021

81. Westin VZ, Schulin M, Hesser H, et al. Acceptance and commitment therapy versus tinnitus retraining therapy in the treatment of tinnitus: a randomised controlled trial. Behav Res Ther. 2011;49:737-747. doi:10.1016/j.brat.2011.08.001 
82. Abbott JA, Kaldo V, Klein B, et al. A cluster randomised trial of an internet-based intervention program for tinnitus distress in an industrial setting. Cogn Behav Ther. 2009;38:162-173. doi:10. 1080/16506070902763174

83. Wilson PH, Henry J, Bowen M, Haralambous G. Tinnitus reaction questionnaire: psychometric properties of a measure of distress associated with tinnitus. J Speech Hear Res. 1991;34:197-201.

84. Henry JL, Wilson PH. An evaluation of two types of cognitive intervention in the management of chronic tinnitus. Scand J Behav Ther. 1998;27:156-166. doi:10.1080/02845719808408510

85. Nyenhuis N, Golm D, Kroner-Herwig B. A systematic review and metaanalysis on the efficacy of self-help interventions in tinnitus. Cogn Behav Ther. 2013;42:159-169. doi:10.1080/16506073.2013.803496

86. Kaldo V, Levin S, Widarsson J, et al. Internet versus group cognitive-behavioral treatment of distress associated with tinnitus: a randomized controlled trial. Behav Ther. 2008;39:348-359. doi:10.1016/j.beth.2007.10.003

87. Konzag TA, Rubler D, Bloching M, et al. [Counselling versus a self-help manual for tinnitus outpatients: a comparison of effectiveness]. Hno. 2006;54:599-604. doi:10.1007/s00106-005-1350-y

88. Malouff JM, Noble W, Schutte NS, Bhullar N. The effectiveness of bibliotherapy in alleviating tinnitus-related distress. J Psychosom Res. 2010;68:245-251. doi:10.1016/j.jpsychores.2009.07.023

89. Nyenhuis N, Zastrutzki S, Weise C, Jager B, Kroner-Herwig B. The efficacy of minimal contact interventions for acute tinnitus: a randomised controlled study. Cogn Behav Ther. 2013;42:127-138. doi:10.1080/16506073.2012.655305

90. Jastreboff PJ, Hazell JW. Tinnitus Retraining Therapy: Implementing the Neurophysiological Model. UK: Cambridge University Press; 2004.

91. Higgins JPT, Green S. Cochrane Handbook for Systematic Reviews of Interventions Version 5.1.0. London, UK: The Cochrane Collaboration; 2011.

92. Zenner HP, Vonthein R, Zenner B, et al. Standardized tinnitusspecific individual cognitive-behavioral therapy: a controlled outcome study with 286 tinnitus patients. Hear Res. 2013;298:117125. doi:10.1016/j.heares.2012.11.013

93. Andersson G, Kaldo V. Internet-based cognitive behavioral therapy for tinnitus. J Clin Psychol. 2004;60:171-178. doi:10.1002/jclp.10243

94. Cima RF, Maes IH, Joore MA, et al. Specialised treatment based on cognitive behaviour therapy versus usual care for tinnitus: a randomised controlled trial. Lancet. 2012;379:1951-1959. doi:10.1016/ S0140-6736(12)60469-3

95. Feeny D, Furlong W, Torrance GW, et al. Multiattribute and singleattribute utility functions for the health utilities index mark 3 system. Med Care. 2002;40:113-128. doi:10.1097/00005650200202000-00006

96. Beukes EW, Andersson G, Allen PM, Manchaiah V, Baguley DM. Effectiveness of guided internet-based cognitive behavioral therapy vs face-to-face clinical care for treatment of tinnitus: a randomized clinical trial. JAMA Otolaryngol Head Neck Surg. 2018;144:11261133. doi:10.1001/jamaoto.2018.2238
97. Beukes EW, Baguley DM, Allen PM, Manchaiah V, Andersson G. Audiologist-guided internet-based cognitive behavior therapy for adults with tinnitus in the United Kingdom: a randomized controlled trial. Ear Hear. 2018;39:423-433. doi:10.1097/AUD.000 0000000000505

98. Meikle MB, Henry JA, Griest SE, et al. The tinnitus functional index: development of a new clinical measure for chronic, intrusive tinnitus. Ear Hear. 2012;33:153-176. doi:10.1097/AUD.0b013e31 $822 \mathrm{f} 67 \mathrm{c} 0$

99. Aazh H, Moore BCJ. Incidence of discomfort during pure-tone audiometry and measurement of uncomfortable loudness levels among People seeking help for tinnitus and/or hyperacusis. Am J Audiol. 2017;26:226-232. doi:10.1044/2017_AJA-17-0011

100. Rogers C. Client-Centered Therapy. New York: Houghton Mifflin; 1965.

101. Wells A. Cognitive Therapy of Anxiety Disorders: A Practice Manual and Conceptual Guide. Chichester, UK: John Wiley \& Sons Inc; 1997.

102. Aazh H. Feasibility of conducting a randomised controlled trial to evaluate the effect of motivational interviewing on hearing-aid use. Int J Audiol. 2016;55:149-156. doi:10.3109/14992027.2015.1074 733

103. Aazh H. Patients' experience of motivational interviewing for hearing aid use: a qualitative study embedded within a pilot randomised controlled trial. $J$ Phonetics Audiology. 2016;2:1-13. doi:10.4172/2471-9455.1000110

104. Miller WR, Rollnick S. Motivational Interviewing: Helping People Change. New York, London: The Guilford Press; 2013.

105. Schulz KF, Altman DG, Moher D. CONSORT 2010 statement: updated guidelines for reporting parallel group randomised trials. BMC Med. 2010;8:18. doi:10.1186/1741-7015-8-18

106. Pocock SJ. Clinical Trials: A Practical Approach. UK: Wiley Chichester; 1983.

107. Cohen J. Statistical Power Analysis for the Behavioral Sciences. New Jersey: Lawrenced Erlbaum Associates, Inc. Publishers; 1988.

108. Cavanna AE, Seri S. Misophonia: current perspectives. Neuropsychiatr Dis Treat. 2015;11:2117-2123. doi:10.2147/NDT. $\mathrm{S} 81438$

109. Reid AM, Guzick AG, Gernand A, Olsen B. Intensive cognitivebehavioral therapy for comorbid misophonic and obsessive-compulsive symptoms: a systematic case study. $J$ Obsessive Compuls Relat Disord. 2016;10:1-9. doi:10.1016/j.jocrd.2016.04.009

110. Dozier TH. Counterconditioning treatment for misophonia. Clin Case Stud. 2015;14:374-387. doi:10.1177/1534650114566924

111. Dozier TH. Treating the initial physical reflex of misophonia with the neural repatterning technique: a counterconditioning procedure. Psychol Thought. 2015;8:189-210. doi:10.5964/psyct.v8i2.138

112. American Psychiatric Association. Diagnostic and Statistical Manual of Mental Disorders: DSM-IV-TR®. American Psychiatric Pub; 2000.
Psychology Research and Behavior Management

\section{Publish your work in this journal}

Psychology Research and Behavior Management is an international, peer-reviewed, open access journal focusing on the science of psychology and its application in behavior management to develop improved outcomes in the clinical, educational, sports and business arenas. Specific topics covered in the journal include: Neuroscience, memory and decision making; Behavior modification and management; Clinical applications; Business and sports performance management; Social and developmental studies; Animal studies. The manuscript management system is completely online and includes a very quick and fair peer-review system, which is all easy to use. Visit http://www. dovepress.com/testimonials.php to read real quotes from published authors. 